\title{
How intrinsic dynamics and coupling architecture interact to generate bursting dynamics in a model network of respiratory conditional pacemakers Christopher Gaiteri ${ }^{1}$ and Jonathan Rubin*2
}

\author{
Address: ${ }^{1}$ Center for Neuroscience, University of Pittsburgh, Pittsburgh, PA, USA and ${ }^{2}$ Department of Mathematics, University of Pittsburgh, \\ Pittsburgh, PA, USA \\ Email: Jonathan Rubin* - rubin@math.pitt.edu \\ * Corresponding author
}

from Sixteenth Annual Computational Neuroscience Meeting: CNS*2007

Toronto, Canada. $7-12$ July 2007

Published: 6 July 2007

BMC Neuroscience 2007, 8(Suppl 2):PI84 doi:I0.I I86/147/-2202-8-S2-PI84

(C) 2007 Gaiteri and Rubin; licensee BioMed Central Ltd.

Experiments suggest that the generation of robust, synchronized bursting within the pre-Bötzinger complex (pre-BötC) of the mammalian brainstem may be critical for respiration, particularly in low oxygen states. The intrinsic dynamics of individual respiratory cells within the pre-BötC, in the absence of coupling, varies widely, with some cells exhibiting tonic spiking, others generating rhythmic bursting, and still others remaining predominantly quiescent. How such a heterogeneous population can produce highly adaptable synchronized bursting remains an open question.

We explored how the distribution of cell types, together with the details of the coupling architecture, shape dynamics in a model pre-BötC network. Dynamics of individual cells were represented using a model developed previously, based on experimental data from preBötC recordings [1]. Heterogeity was introduced by selecting parameter values from distributions; however, we controlled the proportions of each cell type present in each simulation. We focused on three different coupling architectures, namely nearest-neighbor, random, and smallworld, representing extremes of order and disorder as well as a neuronally relevant intermediate case. In small-world simulations, we manipulated the intrinsic dynamics of the cells at nodes involved in long-range interactions. To detect bursting in these networks, given the complicated time course of the overall synaptic input to each cell, we developed a novel algorithm based on our knowledge of the dynamical mechanisms underlying the bursting behavior in the pre-BötC model [2].

Our algorithm was able to distinguish between epochs of bursting driven by inactivation of inward currents and epochs of tonic spiking that were abruptly interrupted by withdrawal of inputs. Although networks consisting solely of intrinsic bursters can generate synchronized bursting, the presence of intrinsically quiescent and intrinsically tonic cells was found to enhance the coherence of bursting across the network and the adaptability of bursting. Under appropriate conditions, tonic cells can promote network activity, which can be sculpted into bursting through the dynamics of other cells in the network. Quiescent cells can lead the termination of activity, ensuring that pauses occur between bursts (see also [3]), and can relatively cleanly transmit activity produced by other cells, promoting synchronization. Intriguingly, the most effective burst generation within small-world networks occurred in those with a mixture of quiescent and tonic cells, rather than bursters, at the long-range nodes.

\section{References}

I. Butera RJ, Rinzel J, Smith JC: Models of respiratory rhythm generation in the pre-Bötzinger complex. I. Bursting pacemaker neurons. J Neurophysiol 1999, 82:382-397.

2. Best J, Borisyuk A, Rubin J, Terman D, Wechselberger M: The dynamic range of bursting in a model respiratory pacemaker network. SIAM J Appl Dyn Sys 2005, 4: I I07-I I39. 
3. Rubin J: Bursting induced by excitatory synaptic coupling in non-identical conditional relaxation oscillators or squarewave bursters. Phys Rev E 2006, 74:021917.

Publish with Bio Med Central and every scientist can read your work free of charge

"BioMed Central will be the most significant development for disseminating the results of biomedical research in our lifetime. " Sir Paul Nurse, Cancer Research UK

Your research papers will be:

- available free of charge to the entire biomedical community

- peer reviewed and published immediately upon acceptance

- cited in PubMed and archived on PubMed Central

- yours - you keep the copyright

Submit your manuscript here:

http://www.biomedcentral.com/info/publishing_adv.asp 